\title{
Phytoplankton Dynamics: from the Behavior of Cells to a Transport Equation
}

\author{
R. Rudnicki ${ }^{a, b 1}$ and R. Wieczorek ${ }^{c}$ \\ ${ }^{a}$ Institute of Mathematics, Polish Academy of Sciences, Bankowa 14, 40-007 Katowice, Poland \\ ${ }^{b}$ Institute of Mathematics, Silesian University, Bankowa 14, 40-007 Katowice, Poland \\ ${ }^{c}$ Institute of Mathematics, Polish Academy of Sciences, Bankowa 14, 40-007 Katowice, Poland
}

\begin{abstract}
We present models of the dynamics of phytoplankton aggregates. We start with an individual-based model in which aggregates can grow, divide, joint and move randomly. Passing to infinity with the number of individuals, we obtain a model which describes the space-size distribution of aggregates. The density distribution function satisfies a non-linear transport equation, which contains terms responsible for the growth of phytoplankton aggregates, their fragmentation, coagulation, and diffusion.
\end{abstract}

Key words: phytoplankton dynamics, formation of aggregates, coagulation, fragmentation, diffusion

AMS subject classification: 47J35, 60K35, 92D40

\section{Introduction}

Phytoplankton cells form aggregates - groups of phytoplankton cells living together. Aggregates are dispersed in the water column as a result of currents and turbulence, leading to a patchy distribution of phytoplankton. Phytoplankton is the basis for the vast majority of oceanic and freshwater food chains. In particular, phytoplankton cells are the only food available to the early larval stages of many fish species. At such stages, larvae are passive and can only eat the prey passing in a very close vicinity. The best situation is when the larva is near a phytoplankton aggregate, while on the other hand larvae which stay far from aggregates are not likely to survive. That is why the description of the space and size distribution of phytoplankton aggregates is an important question

\footnotetext{
${ }^{1}$ Corresponding author. E-mail: rudnicki@us.edu.pl
} 
in the study of fish recruitment. There are many different methods of modelling of space or size structure of phytoplankton. One approach works with approximations of densities by empirical concentrations of particles. These models are known to ecologists as advection-diffusion-reaction models [45] and are heavily used in simulations [44]. Some simple diffusion models [33] and [23] describe the vertical distribution of phytoplankton but do not provide us information of the horizontal distribution. Another approach is based on individual behaviour of phytoplankton cells. In this approach particles move randomly under the action of currents and have at random times the ability of dividing into two new particles. This approach leads to the so-called superprocesses, for which we may refer for example to $[2,17,18]$. Such models are called individual-based models.

In this paper we also consider an individual-based model but the individual unit is an aggregate. The model is similar to that considered in [41] but more general and we will concentrate on biological and physical aspects of processes whereas the paper [41] is mainly devoted to mathematical properties of the model. In the model aggregates are structured by size (i.e. their mass which is proportional to the number of cells) and location in the water. The division or death of the individual cells change the size of aggregates. Apart from growth due to cell division within an aggregate, two main mechanisms are at work: splitting of a given aggregate into parts, which is called fragmentation process, and coagulation (aggregation), by which two distinct aggregates join together to form a single one. The main role in the process of coagulation of phytoplankton is played by TEP (Transparent Exopolymer Particles). TEP are by-product of the growth of phytoplankton and their stickiness cause that cells will remain together upon contact (cf. $[11,25,38]$ ). On the other hand the low level of concentration of TEP leads to fragmentation of phytoplankton aggregates. Again, we assume that coagulation is a binary process. It should be mentioned here that our description of the coagulation process is rather simple. We assume that two distinct aggregates join together with some probability, which depends only on the size and the distance between the aggregates. The coagulation is a complex physical process (cf. [11, 25]) including turbulent shear, particle settling and Brownian motion. Also porosity of aggregates and their stickiness play important role in this process [26]. In our model all above mentioned factors are hidden in the probability of aggregation, which makes mathematics much simpler. Our coagulation process has a non-local character. It means that not only aggregates which have the some locations can join together, which makes our model different from the classical Smoluchowski model [42]. Aggregates are moving randomly in the water and the rate of diffusion depends on their size. Diffusion of aggregates, called also turbulent mixing, is a complex process but it can be a crucial factor in phytoplankton dynamics [23].

The main aim of our paper is to describe the relationship between individual based models and a transport equation on the space-size distribution of aggregates. In the paper we present two individual based models. The first is a discrete model, where an aggregate is composed of an integer number of cells and it moves randomly with constant steps on a lattice. Such a model can be useful in computer simulations. In Section 6. we present some numerical simulations based on this model. Using the Clark-Evans index [10] we show that phytoplankton forms a clustered pattern of distribution. The aim of this simulation is to show significant influence of the diffusion on the clustering. The second model is a continuous model where the mass of an aggregate is a positive real number and aggregates are moving like Brownian particles. Whereas the discrete model is 
useful in simulations, the continuous one has interesting connections with the transport equation on the space-size distribution of aggregates (see Remark 6). Usually the limit passage in both models, when the initial numbers of aggregates goes to infinity and the mass of a single cell tends to zero, is a stochastic process with values in the space of measures called a superprocess (see [12, $15,19,2,17,34])$. The measures which are values of this superprocess describe the distribution of particles in space. In our models we also obtain a limit but here this limit is deterministic. Similar approach was applied to a model of coagulation with diffusion by Norris [35]. Measure-valued limits of interacting particle systems leading to the so-called generalized Smoluchowski equations were also considered in $[8,16,24,27,43]$. For the biological models that use similar methods we also refer to [13, 28, 34, 37].

In our model the density of the space and size distribution of particles is a deterministic function, which satisfies a non-linear transport equation, which contains terms responsible for the growth of phytoplankton aggregates, their fragmentation, coagulation, and diffusion. This equation generalizes the model considered in [6]. Similar equations are used in the completely different context of polymerization/depolymerization of chemical particles (cf. $[1,7,9,36,46])$. Other results concerning this subject can be found in $[4,20,30]$ and the papers quoted there.

\section{Discrete individual-based model}

We construct an individual-based model of phytoplankton. In our model an individual is an aggregate that consists of indistinguishable cells with equal masses joined by an organic glue. Each aggregate is described by two variables: its position $x$ in the space $\mathbb{R}^{d}$ and its mass $m$. In this model we assume that a single cell has the mass one, so $m$ is the number of cells in the aggregate. Cells in the aggregate may die or divide into two daughter cells which causes the decrease or growth of the aggregate. The aggregate may shatter into two smaller aggregates or die (sink or be eaten). Thus the whole situation is described by the following processes:

- A single cell in the aggregate may die in a unit of time with probability $\lambda_{m}(m)$ depending on the mass (number of cells) $m$ of the aggregate or may divide leaving two new cells with probability $\lambda_{b}(m)$.

- The whole aggregate moves according to the $\varepsilon$-random walk-i.e. it skips by a vector of the length $\varepsilon$ in one of $2 d$ directions (parallel to one of the axes, $d$ is the dimension of the space) with probability $\left(1 / \varepsilon^{2}\right) D(m) \Delta t$ in the time interval $\Delta t$ (where $D$ is the coefficient depending on the mass).

- The aggregate may die in a unit of time with probability $\lambda_{d}(m)$.

- The aggregate of mass $m$ may split in a unit of time with probability $\lambda_{f}(m)$ into two parts with mass $m_{1}$ and $m_{2}$, where $m_{1}+m_{2}=m$, with probability $p\left(m, m_{1}\right)$ (where $\left.\sum_{m_{1}=1}^{m-1} p\left(m, m_{1}\right)=1\right)$.

- The $i$-th and $j$-th aggregates may join up with probability $\kappa_{i j}$ depending on their mass and locations, and on the state of the whole population. 
The next problem is the position of new aggregates after fragmentation and coagulation. In [41] it is assumed that after fragmentation both new aggregates appear at the same location as their parent. Now we consider a more general model in which after the break up of an aggregate with the center $y$, the new two parts with mass $m_{1}$ and $m_{2}$ have centers at positions $x_{1}$ and $x_{2}$ distributed with a density $a_{f}\left(x_{1}, x_{2}, y, m_{1}, m_{2}\right)$. It means that $a_{f}\left(x_{1}, x_{2}, y, m_{1}, m_{2}\right)$ is a nonnegative function such that

$$
\int_{\mathbb{R}^{d}} \int_{\mathbb{R}^{d}} a_{f}\left(x_{1}, x_{2}, y, m_{1}, m_{2}\right) d x_{1} d x_{2}=1
$$

for $y \in \mathbb{R}^{d}$ and for positive integers $m_{1}, m_{2}$. Furthermore, we assume that $a_{f}$ is symmetrical, i.e.

$$
a_{f}\left(x_{1}, x_{2}, y, m_{1}, m_{2}\right)=a_{f}\left(x_{2}, x_{1}, y, m_{2}, m_{1}\right)
$$

for $x_{1}, x_{2}, y \in \mathbb{R}^{d}$ and for positive integer $m_{1}, m_{2}$.

Coagulation is described by two values: the probability coefficient of coagulation $\kappa$ and the place $\widehat{x}=\widehat{x}\left(x_{1}, x_{2}, m_{1}, m_{2}\right)$ where the new aggregate appears after coagulation of $\left(x_{1}, m_{1}\right)$ and $\left(x_{2}, m_{2}\right)$. Let the ability of the $i$-th aggregate to coagulate be $c\left(m_{i}\right)$, the conditional probability that it joins to the $j$-th aggregate is given by $c\left(m_{j}\right) / \sum_{k=1}^{N} c\left(m_{k}\right)$ and it is modified by a distancedepending coefficient $v\left(x_{i}-x_{j}\right)$. Thus the $i$-th and $j$-th aggregates may join up with probability

$$
\kappa_{i j}=\frac{c\left(m_{i}\right) c\left(m_{j}\right)}{\sum_{k} c\left(m_{k}\right)} v\left(x_{i}-x_{j}\right)
$$

where the sum in the denominator extends over all living individuals.

The term $\kappa$ describing probability of coagulation is significantly different from standard physical models where the probability of coagulation is proportional to the square of the number of particles. We consider the more biologically justifiable case, when the coagulation ability of a single aggregate is not unbounded, but approximately constant. The coagulation ability depends on the concentration of some organic glue (TEP) [11, 38]. It means that the probability of joining is a function of production of TEP by an aggregate, which depends on the mass of the aggregate. It should be noted that the function $\kappa$ is symmetrical, i.e. it does not depend on the order of aggregates.

From the mathematical point of view $\widehat{x}$ may be given arbitrarily. In the theory of fragmentationcoagulation processes many different types of coagulation are considered [3, 14, 32, 42]. Although there are many applications of the coagulation theory to description of aggregation of phytoplankton cells $[11,25,26,38]$, the mechanism by which aggregates are formed is not fully understood. That is why we suggest some particular forms of $\widehat{x}$ remaining, at the same time, rather general. It is natural to assume that the aggregate after coagulation should appear somewhere between its parent aggregates. That is why there are essentially two forms of $\widehat{x}$. The simpler one is considered in [41], where we assume that the new aggregate appears exactly in the half way between the two old ones, namely

$$
\widehat{x}\left(x_{1}, x_{2}\right)=\frac{x_{1}+x_{2}}{2} .
$$

A little more complicated (but physically more justifiable) is $\widehat{x}$ of the form

$$
\widehat{x}\left(x_{1}, x_{2}, m_{1}, m_{2}\right)=\frac{m_{1} x_{1}+m_{2} x_{2}}{m_{1}+m_{2}},
$$


which describes the case when the new aggregate appears at the center of mass of the two previous ones. In this paper we consider a non-local form of coagulation. The location $x$ of an aggregate after coagulation of two aggregates with the mass $m_{1}, m_{2}$ and the positions $y_{1}, y_{2}$, respectively, is given by a density function $a_{c}\left(x ; y_{1}, y_{2}, m_{1}, m_{2}\right)$.

It should be noted that our model of coagulation and fragmentation differs from the classical Smoluchowski local model [42] in which parents and descendant aggregates are located at the same place. Nonlocal models similar to ours appear in [29], [30]. The local descriptions of fragmentation and coagulation processes can be obtained from nonlocal ones by proper limit passages [31]. In our case we define

$$
a_{c}^{\varepsilon}\left(x ; y_{1}, y_{2}, m_{1}, m_{2}\right)=\varepsilon^{-d} \varphi\left(\frac{x-\widehat{x}}{\varepsilon}\right),
$$

where $\varphi$ is a smooth radially symmetric density function. If $\varepsilon \rightarrow 0$ then $a_{c}^{\varepsilon}$ goes to a singular measure corresponding to the deterministic location $\widehat{x}=\widehat{x}\left(y_{1}, y_{2}, m_{1}, m_{2}\right)$, for example $\widehat{x}$ can be given by one of the formulae (2.2) and (2.3). In a similar manner we can describe fragmentation processes which have a local character. For example, if we take the function $a_{f}^{\epsilon}$ of the form

$$
a_{f}^{\varepsilon}\left(x_{1}, x_{2}, y, m_{1}, m_{2}\right)=\varepsilon^{-2 d} \varphi\left(\frac{x_{1}-y}{\varepsilon}\right) \varphi\left(\frac{x_{2}-y}{\varepsilon}\right),
$$

and we go with $\varepsilon$ to zero, we receive the model where after fragmentation both new aggregates appear at the same location as the parent. Analogously, if $\varphi$ and $\psi$ are smooth radially symmetric density functions and

$$
a_{f}^{\varepsilon}\left(x_{1}, x_{2}, y, m_{1}, m_{2}\right)=\varepsilon^{-d} \psi\left(x_{1}-y\right) \varphi\left(\frac{m_{1} x_{1}+m_{2} x_{2}-\left(m_{1}+m_{2}\right) y}{\varepsilon m_{2}}\right),
$$

then in the limit $\varepsilon \rightarrow 0$ we obtain a fragmentation model with the conservation of the center of mass. The density function $\psi$ describes the space distribution of the first aggregate.

Remark 1. The model presented in this section is, in fact, a "hybrid" one because the position of an aggregate is a continuous variable whereas its mass is a discrete value. It is not difficult to change this model to an entirely discrete one by replacing the space of positions $\mathbb{R}^{d}$ by the lattice $\mathbb{Z}^{d}$. One should also replace continuous functions which appear in the processes of fragmentation and coagulation by their discrete versions and, in fact, we have done so in simulations.

\section{The limit passage}

The state of our model is described by the vector $\left(k ; x_{1}, m_{1}, \ldots, x_{k}, m_{k}\right)$, where $k$ is the number of aggregates and $x_{i}, m_{i}$, for $i=1, \ldots, k$, denote, respectively, the location and mass of the $i$ th aggregate. Since $k$ (and so the number of variables) changes during evolution, and the order of pairs $x_{i}, m_{i}$ is not important, we need a special state space. We use the set of measures

$$
\mathcal{N}=\left\{\sum_{i=1}^{k} \delta_{x_{i}, m_{i}}: k \in \mathbb{N},\left(x_{i}, m_{i}\right) \in \mathbb{R}^{d} \times \mathbb{N}\right\},
$$


i.e. we denote the aggregate of size $m$ at position $x$ by the Dirac delta measure $\delta_{x, m}$ at $(x, m) \in$ $\mathbb{R}^{d} \times \mathbb{N}$. The set $\mathcal{N}$ is a subspace of the space $\mathcal{M}$ of all finite Borel measures on $\mathbb{R}^{d} \times \mathbb{R}^{+}$with the topology of weak convergence.

The model of the phytoplankton dynamics given in the previous section can be described by means of a stochastic process $\nu(t)$ with values in the space $\mathcal{N}$. The process $\nu(t)$ is a jump process and the formal proof of its existence is standard. The precise formulation and the proof in a special case can be found in [41].

Now, we construct the sequence of rescaled processes $\left\{\nu^{(N)}(t)\right\}_{t \geq 0}, N \in \mathbb{N}$, based on $\nu(t)$ that will approximate some continuous model. Assume that the number of particles at time 0 increases to infinity as $N \rightarrow \infty$ and assume that the mass of each cell is $1 / N$. The $N$-th process $\nu^{(N)}$ has values in the space

$$
\mathcal{N}^{N}=\left\{\frac{1}{N} \sum_{i=1}^{k} \delta_{x_{i}, n_{i} / N}: k \in \mathbb{N},\left(x_{i}, \frac{n_{i}}{N}\right) \in \mathbb{R}^{d} \times \frac{1}{N} \mathbb{N}\right\} .
$$

The rescaling means that the process $N \nu^{(N)}(t)$ behaves like $\nu(t)$ with appropriate coefficients. Namely:

- The birth or death of the cell means the change of mass by a factor of $1 / N$.

- We set the step of the random walk to be $\varepsilon=1 / N$.

- The result of fragmentation of an aggregate of size $m=n / N$ may have any mass in $1 / N, 2 / N, \ldots,(n-1) / N$; so we assume that coefficients $p^{(N)}$ are such that

$$
\sum_{\bar{n}=1}^{n-1} p^{(N)}(n / N, \bar{n} / N)=1
$$

moreover, we assume that there exists a continuous function $q: \mathbb{R}^{+} \times \mathbb{R}^{+} \rightarrow \mathbb{R}^{+}$such that for all $m, \bar{m} \in \mathbb{R}^{+}, \bar{m} \leq m$ and all sequences $\left(n_{N}\right),\left(\bar{n}_{N}\right)$ of positive integers such that $n_{N} / N \rightarrow m$ and $\bar{n}_{N} / N \rightarrow \bar{m}$ as $N \rightarrow \infty$ we have $N p^{(N)}\left(n_{N} / N, \bar{n}_{N} / N\right) \rightarrow q(m, \bar{m})$ and this convergence is uniform,

- the coagulation term remains unchanged.

Notice that the function $q$ satisfies $\int_{0}^{m} q(m, \bar{m}) d \bar{m}=1$ for $m>0$ and $q(m, \bar{m})=q(m, m-\bar{m})$ for all $m>\bar{m}>0$. The probabilistic kernel $P(m, A):=\int_{A} q(m, \bar{m}) d \bar{m}$ will describe the distribution of the size of the aggregates after fragmentation if the aggregate before fragmentation has size $m$. The condition $N p^{(N)}\left(n_{N} / N, \bar{n}_{N} / N\right) \rightarrow q(m, \bar{m})$ can be rewritten in the form

$$
p^{(N)}(m, \bar{m}) \approx \int_{\bar{m}-1 / N}^{\bar{m}} q(m, r) d r .
$$

Condition (3.1) means that the discrete distribution $p^{(N)}$ with the step $1 / N$ approximates the continuous distribution density $q$. 
We assume that the functions $D, \lambda_{f}, \lambda_{d}, c, v, a_{f}$ and $a_{c}$ are bounded and continuous; moreover $c(m)>0$ for all $m \in[0, \infty)$. We also assume that the functions $\lambda_{m}$ and $\lambda_{b}$ are continuous and the function $\lambda(m)=m\left(\lambda_{b}(m)-\lambda_{m}(m)\right)$ is bounded from above. The last assumption seems to be natural because the function $\lambda$ is the growth rate of an aggregate. For example $\lambda_{m}$ can be a constant function and $\lambda_{b}$ can be of the form $\lambda_{b}(m)=b_{1} /\left(b_{2}+m\right)$.

The mentioned above functions coincide with the functions used in the formulation of the discrete model for the integer mass.

Measures which are values of the process $\nu^{(N)}(t)$ can be interpreted in the following way. Let $A$ be a subset of $\mathbb{R}^{d}$ and $\left[m_{1}, m_{2}\right]$ be an interval in $\mathbb{R}^{+}$. Then for any time $t \geq 0$ and any trajectory $N \nu^{(N)}(t)\left(A \times\left[m_{1}, m_{2}\right]\right)$ is the number of aggregates located in the set $A$ with mass from the interval $\left[m_{1}, m_{2}\right]$.

Consider the limit passage when the initial number of aggregates tends to infinity as $N \rightarrow \infty$ and the mass of a single cell tends to zero $m=1 / N \rightarrow 0$.

Theorem 2. We assume that the initial distribution of the process $\nu^{N}(0)$ converges weakly to some finite measure $\nu^{\infty}(0)$ on $\mathbb{R}^{d} \times \mathbb{R}^{+}$. Then the sequence of processes $\nu^{N}(t)$ converges in distribution to a (non-random) function $\nu^{\infty}(t)$ with values in the space of measures.

Remark 3. The formal definition of the convergence of $\nu^{N}(t)$ in distribution is given in Appendix. Some properties of the function $\nu^{\infty}(t)$ are discussed in Section 5.

The proof of Theorem 2 is based on [22, Corollary 8.16, Chapter 4] and its scheme is following. Firstly we check that the processes $\nu_{N}(t)$ solve some martingale problems. Then we define an operator $\mathcal{L}$ corresponding to the limit process. Next we prove that if some process solves the $\mathcal{L}-$ martingale problem then it is deterministic and unique. Then we check that the sequence $\nu^{(N)}$ converges to the solution of this problem. The detailed proof in a special case can be found in [41]. This proof can be adapted to the general case without any difficulties.

\section{Continuous individual-based model}

Instead of the discrete individual model from Section 2. we can consider a continuous model in which the mass of an aggregate is a positive number and aggregates are moving like Brownian particles in the space $\mathbb{R}^{d}$. In this model an individual is described by two variables $(x, m)$, where $x \in \mathbb{R}^{d}$ is its position in space and $m>0$ is its mass. As before, aggregates grow as a result of divisions of cells, may die, break up and joint up. The whole process is described as follows.

- The growth rate is $\lambda(m)=\left(\lambda_{b}(m)-\lambda_{m}(m)\right) m$. It means that an aggregate grows according to the equation

$$
m^{\prime}=\lambda(m) .
$$

- The aggregate with mass $m$ moves according to the Brownian motion $\sqrt{2 D(m)} W(t)$, where $W(t)$ is the standard Wiener process in $\mathbb{R}^{d}$.

- The aggregate may die in a unit of time with probability $\lambda_{d}(m)$. 
- A fraction $\lambda_{f}(m)$ of aggregates of mass $m$ are undertaking breakup in a unit of time. The sizes $m_{1}$ and $m_{2}$ of the two pieces are described by the conditional density $q\left(m, m_{i}\right), i=1,2$, and their positions $x_{1}$ and $x_{2}$ are distributed with the density $a_{f}\left(x_{1}, x_{2}, y, m_{1}, m_{2}\right)$, where $y$ is the position of the parent aggregate.

- Two aggregates may join up with probability $\kappa$ depending on their mass and locations, and on the state of the whole population. The position of the new aggregate is described by the density $a_{c}$.

Remark 4. Usually, it is assumed that the growth rate $\lambda(m)$ is positive, but we allow for a possibility that $\lambda(m)$ is negative. For example, if we assume that aggregates cannot grow more than some maximum size $m^{*}$, then two large aggregates can joint up and form an aggregate larger than $m^{*}$. Such an aggregate should decrease because the death rate $\lambda_{m}(m)$ of single cells is greater than the division rate $\lambda_{b}(m)$.

Similarly to Section 2. the state of the system can be described by means of a stochastic process $\nu_{*}(t)$ with values in the space

$$
\mathcal{N}_{*}=\left\{\sum_{i=1}^{k} \delta_{x_{i}, m_{i}}: k \in \mathbb{N},\left(x_{i}, m_{i}\right) \in \mathbb{R}^{d} \times \mathbb{R}^{+}\right\} .
$$

The set $\mathcal{N}_{*}$ is a subspace of the space $\mathcal{M}$. Similarly to Section 3. we can construct the sequence of rescaled processes $\left\{\nu_{*}^{(N)}(t)\right\}_{t \geq 0}, N \in \mathbb{N}$, based on $\nu_{*}(t)$ that will approximate some continuous model. The $N$ th process $\nu_{*}^{(N)}$ has values in the space

$$
\mathcal{N}_{*}^{N}=\left\{\frac{1}{N} \sum_{i=1}^{k} \delta_{x_{i}, m_{i}}: k \in \mathbb{N},\left(x_{i}, m_{i}\right) \in \mathbb{R}^{d} \times \mathbb{R}^{+}\right\} .
$$

Consider the limit passage when the initial number of aggregates tends to infinity as $N \rightarrow \infty$.

Theorem 5. We assume that the initial distribution of the process $\nu_{*}^{N}(0)$ converges weakly to some finite measure $\nu^{\infty}(0)$ on $\mathbb{R}^{d} \times \mathbb{R}^{+}$. Then the sequence of processes $\nu_{*}^{N}(t)$ converges in distribution to $\nu^{\infty}(t)$, where $\nu^{\infty}(t)$ is the same non-random measure-valued function as in Theorem 2.

Remark 6. Theorems 2 and 5 can be formulated in a more general setting. Namely, the initial value of the processes $\nu^{N}(0)$ or $\nu_{*}^{N}(0)$ can be random variables with values in the spaces $\mathcal{N}^{N}$ and $\mathcal{N}_{*}^{N}$, respectively. Then if $\nu^{N}(0)$, respectively $\nu_{*}^{N}(0)$, converges almost surely to some finite measure $\nu^{\infty}(0)$ as $N \rightarrow \infty$, then the sequence of processes $\nu^{N}(t)$, respectively $\nu_{*}^{N}(t)$, converges in distribution to $\nu^{\infty}(t)$. Moreover, we have the following interesting relationship between the stochastic process $\nu_{*}^{N}(t)$ and the function $\nu^{\infty}(t)$. Let the measures $\mu_{*}^{N}(t)$ be the mean values of the process $\nu_{*}^{N}(t)$, i.e. $\mu_{*}^{N}(t)$ are measures such that for every Borel subset $A$ of $\mathbb{R}^{d} \times \mathbb{R}^{+}$and $t \geq 0$ we have $\mu_{*}^{N}(t)(A)=\mathrm{E}\left(\nu_{*}^{N}(t)(A)\right)$. If for some $N \in \mathbb{N}$ we have $\nu^{\infty}(0)=\mu^{N}(0)$, then $\nu^{\infty}(t)=\mu^{N}(t)$ for each $t \geq 0$. 


\section{Equation on densities}

Consider the function $\nu^{\infty}(t)$. If $\nu^{\infty}(0)$ is absolutely continuous with respect to the Lebesgue measure on $\mathbb{R}^{d} \times \mathbb{R}^{+}$, then $\nu^{\infty}(t)$ is also absolutely continuous, i.e. there exists a nonnegative measurable function $u$ such that $\nu(t)(d x d m)=u(t, x, m) d x d m$. For each $t \geq 0$ the function $u(t, x, m)$ is the density of the space-size distribution of aggregates. It should be noted that the term "distribution" is not used in the strictly probabilistic sense, i.e. the integral $\int_{\mathbb{R}^{d} \times \mathbb{R}^{+}} u(t, x, m) d x d m$, which means the total number of aggregates, can be different from one and can change in time. One can check that $u$ satisfies the following equation

$$
\frac{\partial u(t, x, m)}{\partial t}=L u(t, x, m)+B u(t, x, m)+F u(t, x, m)+C u(t, x, m),
$$

where the operators $L, B, F$, and $C$ are given by

$$
\begin{aligned}
L f(x, m)= & D(m) \Delta_{x} f(x, m), \\
B f(x, m)= & -\frac{\partial}{\partial m}(\lambda(m) f(x, m)), \\
F f(x, m)= & \int_{\mathbb{R}^{d}} \int_{\mathbb{R}^{d}} \int_{m}^{\infty} f(y, \widetilde{m}) \lambda_{f}(\widetilde{m}) q(\widetilde{m}, m) \\
& \times\left[a_{f}(x, z, y, m, \widetilde{m}-m)+a_{f}(z, x, y, m, \widetilde{m}-m)\right] d \widetilde{m} d y d z \\
& -\lambda_{f}(m) f(x, m)-\lambda_{d}(m) f(x, m), \\
C f(x, m)= & \frac{1}{V(f)}\left[\int_{\mathbb{R}^{d}} \int_{\mathbb{R}^{d}} \int_{0}^{m} 2^{d} a_{c}\left(x ; y_{1}, y_{2}, \bar{m}, m-\bar{m}\right) c(\bar{m}) c(m-\bar{m})\right. \\
& \times v\left(y_{1}-y_{2}\right) f\left(y_{1}, \bar{m}\right) f\left(y_{2}, m-\bar{m}\right) d y_{1} d y_{2} d \bar{m} \\
& \left.-\int_{\mathbb{R}^{d}} \int_{0}^{\infty} 2 c(m) c(\bar{m}) v(x-\bar{x}) f(x, m) f(\bar{x}, \bar{m}) d \bar{m} d \bar{x}\right],
\end{aligned}
$$

where $V(f)=\int_{\mathbb{R}^{d}} \int_{0}^{\infty} c(m) f(x, m) d x d m$ and $\Delta_{x}$ is the Laplace operator with respect to the spatial variable $x$.

We write $u(t)(x, m)$ instead of $u(t, x, m)$ and we consider solutions $u(t)$ of $(5.1)$ as functions from $\mathbb{R}^{+}$with values in the set $X_{+}$, where $X_{+}$is the subset of all nonnegative and nonzero functions from the space $X=L^{1}\left(\mathbb{R}^{d} \times \mathbb{R}^{+}\right)$. Equation (5.1) can be treated as an evolution equation

$$
u^{\prime}(t)=(L+B+F+C) u(t) .
$$

Theorem 7. Let $D(m)>0$ and $c(m)>0$ for $m \geq 0$. For any $u_{0} \in X_{+}$there exists a unique solution $u(t)$ of (5.6) such that $u(0)=u_{0}$.

The proof of Theorem 7 is based on that of Theorem 1 in [6] and goes as follows. Firstly we check that the operator $L+B$ generates a Markov semigroup on $X$. We recall that a Markov operator is a positive linear operator defined on the space $L^{1}$ which preserves the integral and a Markov semigroup is a strongly continuous semigroup of Markov operators. Since $F$ is a bounded 
linear operator on $X$, from the Phillips perturbation theorem it follows that the operator $L+B+F$ generates a strongly continuous semigroup of bounded positive operators on $X$. Then we prove that the operator $C$ is Lipschitzian on $X_{+}$. The rest of the proof is a simple consequence of the method of variation of parameters (see e.g. [39]).

Remark 8. According to Theorem 7, for each initial condition equation (5.1) has a unique solution that exists for all positive time. This feature distinguishes (5.1) from physical coagulation equations which do not have global solutions. This surprising property of (5.1) results from the special form of the coagulation term (5.5), which is homogeneous with respect to $f$.

\section{Numerical simulations}

In this section we present some numerical results based on our first individual-based model. Our aim is to show that in our model phytoplankton aggregates form clustered patterns of distribution. We will study the spatial distribution of phytoplankton by means of the Clark-Evans index [10]. This index compares the observed mean nearest neighbour distance to that expected for a random distribution of individuals. The Clark-Evans index is given by

$$
C E I=\frac{d_{\mathrm{emp}}}{d_{\exp }}
$$

where $d_{\mathrm{emp}}$ and $d_{\text {exp }}$ are, respectively, the empirical and the expected mean distances to the nearest neighbour. If individuals are located at points $x_{1}, x_{2}, \ldots, x_{N}$, then

$$
d_{\mathrm{emp}}=\frac{1}{N} \sum_{i=1}^{N} \min _{j \neq i}\left(\operatorname{dist}\left(x_{i}, x_{j}\right)\right) \text {. }
$$

The expected mean distance $d_{\exp }$ is the expected value of (6.2) if points $x_{1}, x_{2}, \ldots, x_{N}$ are independently and uniformly distributed in some domain $D \subset \mathbb{R}^{d}$. For large values of $N$ we have

$$
d_{\exp } \approx \frac{1}{2} \sqrt{\frac{S}{N}},
$$

where $S$ is the area of the domain $D$. In computation we use the approximate formula (6.3) for $d_{\exp }$. Although CEI measures pairing of elements, it is also a quite good tool for measuring of clustering of real objects. The measuring of the distances from one object to another provides a variable for the measurement of spacing. It is assumed that Clark-Evans index indicates regular, random and clustered patterns of distribution. Namely, if $C E I$ is approximately one then points are randomly distributed. If $C E I>1$ they have regular structure and if $C E I<1$ they form clustered patterns of distribution. Since CEI is simple to calculate it is commonly used in various applications (see e.g. [5, 21, 40]).

Since our aggregates have different sizes we also introduce another index, called the weighted Clark-Evans index $\overline{C E I}$, which, in our opinion, can be more adequate. We define

$$
\overline{C E I}=\frac{\bar{d}_{\mathrm{emp}}}{\bar{d}_{\mathrm{exp}}},
$$


where

$$
\bar{d}_{\mathrm{emp}}=\left(\sum_{i=1}^{N} m_{i} \min _{j \neq i}\left(\operatorname{dist}\left(x_{i}, x_{j}\right)\right)\right) /\left(\sum_{i=1}^{N} m_{i}\right) .
$$

and $\left(x_{1}, m_{1}\right), \ldots,\left(x_{N}, m_{N}\right)$ are locations and mass of individuals. Analogously, we define $\bar{d}_{\text {exp }}$ as the expected value of (6.5) assuming that mass of individuals are independent on their positions. It is easy to check that

$$
\bar{d}_{\exp }=d_{\exp } .
$$

Thus we can use approximate formula for $\bar{d}_{\text {exp }}$ :

$$
\bar{d}_{\exp } \approx \frac{1}{2} \sqrt{\frac{S}{N}} .
$$

The weighted Clark-Evans index also indicates regular, random and clustered patterns of distribution. Namely, if $\overline{C E I}$ is approximately one then aggregates are randomly distributed and if $\overline{C E I}<1$ they form clustered patterns of distribution. But since large aggregates have a crucial influence on the weighted Clark-Evans index we can say more about clustering if we compare both indices. Namely, if $\overline{C E I}<C E I$ then the mean distances for large aggregates to the nearest neighbour is smaller than average for all aggregates, which suggests that clusters are formed around large aggregates.

Now we present results of three numerical experiments which show how the Clark-Evans index and the weighted Clark-Evans index depend on coefficients of diffusion. It should be noted that the parameters chosen in the model have no connection with the real physical processes in the phytoplankton dynamics. The aim of our simulations is rather to show that the process of clustering of phytoplankton aggregates depends significantly on the type of diffusion. We also show that the spatial structure of aggregates can become clustered even if we consider models without coagulation. The reason for such a phenomenon is that the processes of growth and fragmentation can lead to appearing of clusters of relatively large aggregates.

We investigate here the first individual-based model without the coagulation process. The simulations are driven on a torus represented by a net of the size $400 \times 400$. In all experiments we started with 350 individuals randomly located on the net and with random mass. Figures 1-3 present the time evolution of the standard and weighted Clark-Evans indices. The cases with the mass independent diffusion are shown in Fig. 1 (a large diffusion coefficient) and Fig. 2 (a small diffusion coefficient). In both cases the standard and weighted Clark-Evans indices are nearly the same. The only difference is that if a diffusion coefficient is smaller than the indices are smaller, which means that the structure is more clustered, which agrees with the physical intuition. The more interesting case is presented in Fig 3, where the diffusion coefficient decreases if the mass increases. This case corresponds to standard physical models of diffusion. In this case the weighted Clark-Evans index decreases faster than the Clark-Evans index. It means that not only spatial structure becomes clustered but also large aggregates plays crucial rôle in the process of clustering. 


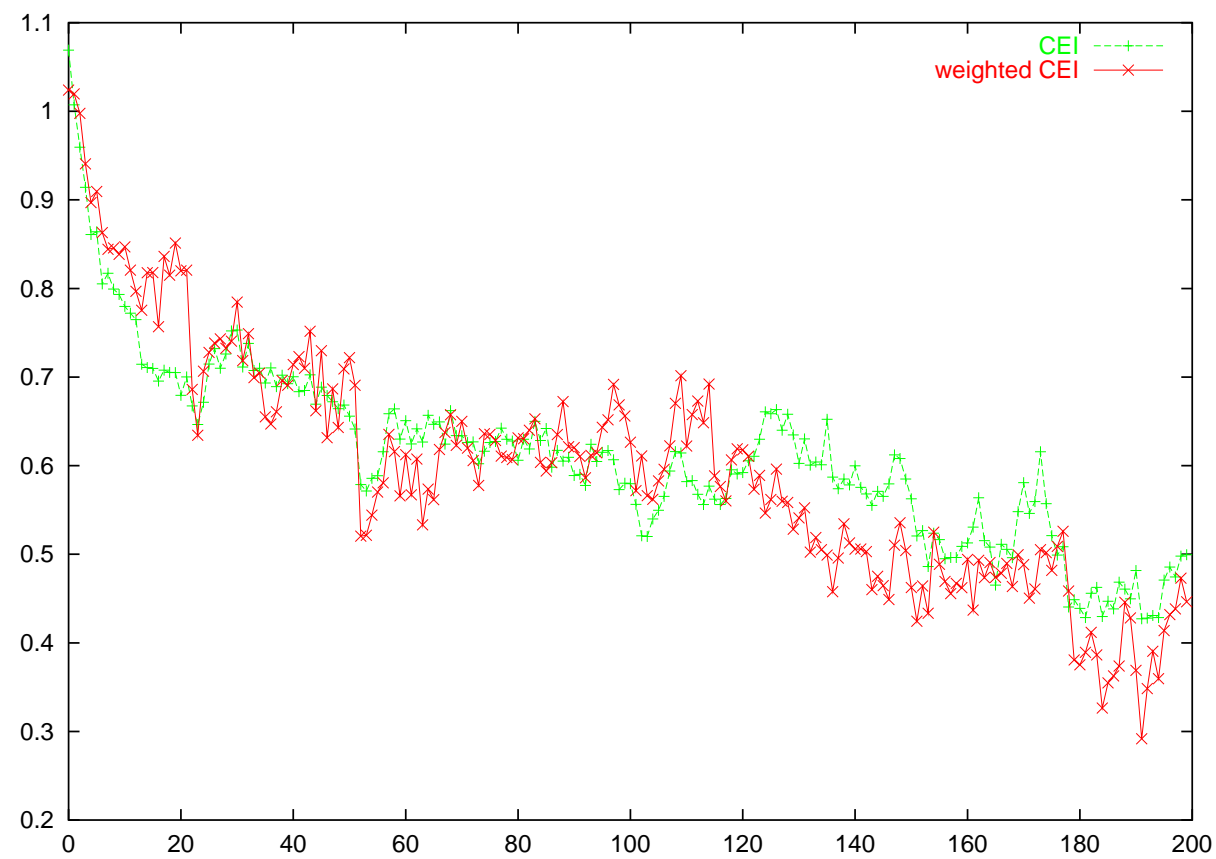

Figure 1: $C E I$ and $\overline{C E I}$ in the case of a constant and large diffusion.

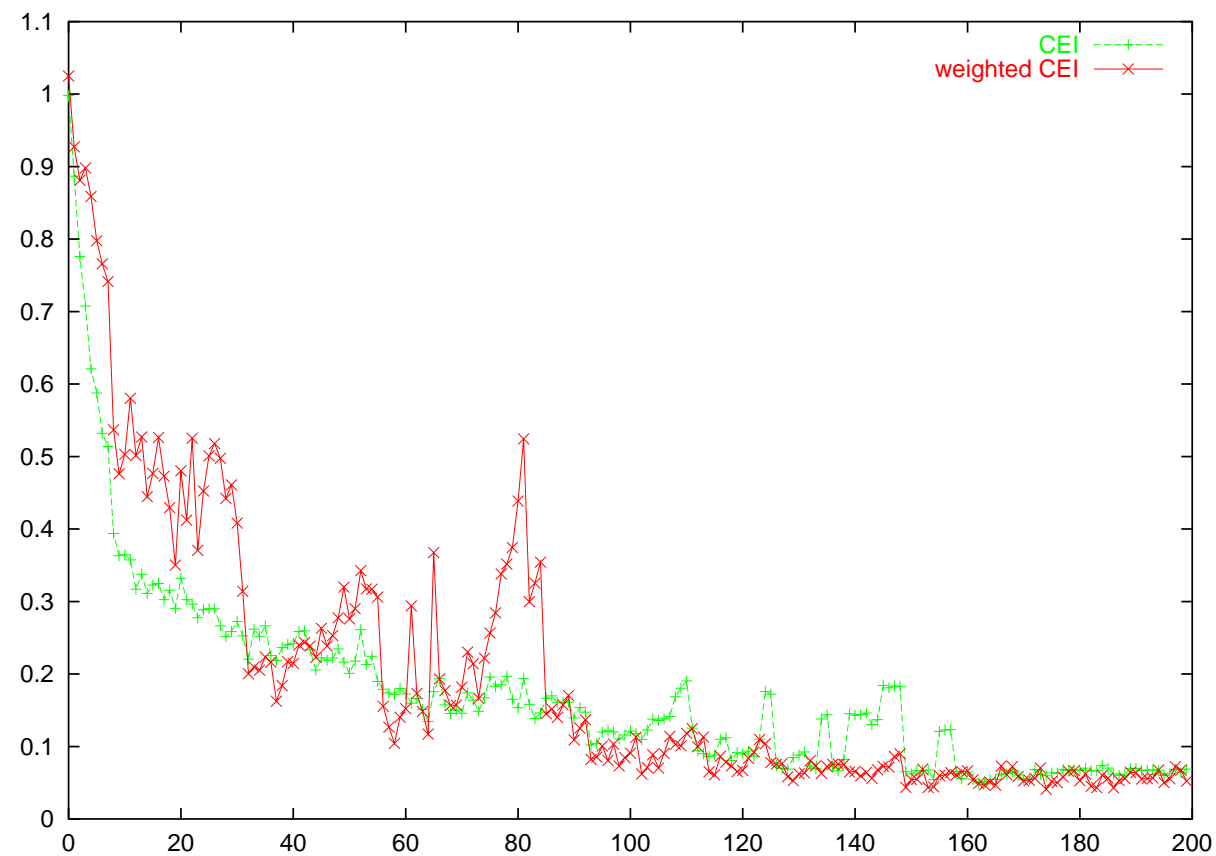

Figure 2: $C E I$ and $\overline{C E I}$ in the case of a constant and small diffusion. 


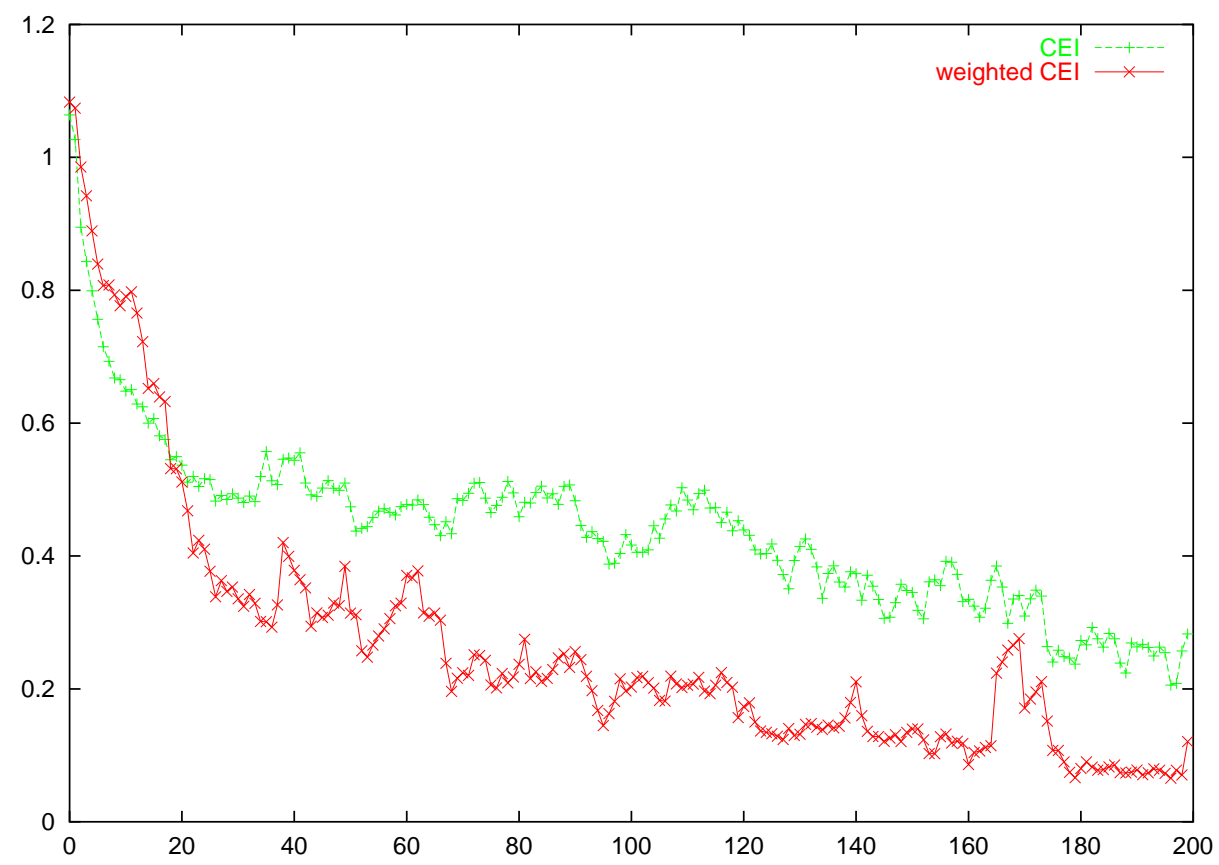

Figure 3: $C E I$ and $\overline{C E I}$ in the case when the diffusion coefficient decreases if the mass increases.

\section{Concluding remarks}

New individual-based models of phytoplankton dynamics have been introduced. In these models individuals are aggregates, which consists of phytoplankton cells. Using the martingale approach, we have presented a limit passage from microscopic description (ibm) to macroscopic one, when the numbers of individuals goes to infinity and the mass of a single cell goes to zero. In our case the limit is a deterministic process and the function describing space and size distribution aggregates satisfies fragmentation-coagulation equation with diffusion (5.1). This equation has the property of the global existence and uniqueness of solutions. Usually, coagulation-fragmentation equations which describe physical processes [36] fail to have global solutions. The global existence of solution of (5.1) is connected with the fact that our coagulation term is different from the classical one [42]. Of course, it would be interesting to investigate models of phytoplankton dynamics where coagulation process is described in the same way as in physical processes. The problem can be here with biological interpretation of the gelation phenomenon which appears in physical models.

To our knowledge, little is known about the behaviour of solutions to equations such as (5.1). A version of this equation without diffusion was studied in [6]. In that paper sufficient conditions were given for existence of large aggregates, which is important from biological point of view. Our equation should preserve this property. The second question is the space distribution of aggregates. In nature phytoplankton cells have patchy distribution. We hope that our model has similar feature. Since it is difficult to study (5.1) analytically, the individual-based model presented in Section 2. serves us as a useful tool for numerical simulations. Numerical investigation of the standard and 
weighted Clark-Evans indices confirms the clustered pattern of the spatial distribution in the case of a small diffusion and if the diffusion decreases with mass. Moreover, in the latter case we check that the weighted Clark-Evans index is significantly smaller than the standard Clark-Evans index, which suggests that clusters are formed around large aggregates. This property is of great importance in survival of early larval stage of fish.

Appearing of the clustered spatial structure in the individual-based model also suggests that the measure describing the distribution of phytoplankton cells and aggregates tends to measures with fractal supports. Such effect appears, for example, in an individual-based model considered in [2], where a superprocess describing the distribution of cells in 3-dimensional space has values in the space of measures with 2-Hausdorff-dimensional support. However, in the model [2], the superprocess was obtained by the limit approach when the lifetime of a cell tends to zero, what is a little strange from biological point of view. In our case the diffusion of large aggregates is small, and such aggregates should establish interesting space structure.

Since in our model the factor that decides on fragmentation and coagulation is an organic glue, it would be interesting to consider a model with immediate coagulation. In this model there is a function $\rho\left(m_{1}, m_{2}\right)$ such that if the distance of two aggregates with mass $m_{1}$ and $m_{2}$ is less than $\rho\left(m_{1}, m_{2}\right)$, then they join together immediately. Of course, in order to exclude immediate coagulation after fragmentation we should modify the fragmentation process. Namely, if twin aggregates have mass $m_{1}, m_{2}$ then the distance between their centers at the moment of birth should be greater than $\rho\left(m_{1}, m_{2}\right)$. Such a model is very interesting and seems to be adequate to our problem but seems not to yield easily to rigorous analysis.

\section{Acknowledgements}

This research was partially supported by the State Committee for Scientific Research (Poland) Grants No. 2 P03A 031 25, N201 047 31/3830 and by EC FP6 Marie Curie ToK programme SPADE2, MTKD-CT-2004-014508 and Polish MNiSW SPB-M.

\section{Appendix}

Now we recall some definitions concerning stochastic processes with values in the space of measures (see [22] for details). The stochastic processes $\nu^{N}$ and $\nu_{*}^{N}$ take values in the space of all finite Borel measures $\mathcal{M}$ on $\mathbb{R}^{d} \times \mathbb{R}^{+}$. The space $\mathcal{M}$ is equipped with the topology of the weak convergence. The space $\mathcal{M}$ is a Polish space in the Prohorov metric. The trajectories of the processes $\nu^{N}$ and $\nu_{*}^{N}$ are càldàg functions, i.e. are right continuous functions with left hand limits. The space of all càldàg functions with the Skorokhod topology is denoted by $D([0, \infty), \mathcal{M})$. The distribution of the process $\nu^{N}(t)$ induces the probabilistic measure $P_{N}$ on the space of trajectories $D([0, \infty), \mathcal{M})$. By the convergence in distribution of the sequence of the processes $\nu^{N}(t)$ we mean the weak convergence of the measures $P_{N}$. 


\section{References}

[1] A.S. Ackleh, K. Deng. On the first order hyperbolic coagulation model. Math. Methods Appl. Sci., 26 (2003), 703-715.

[2] R. Adler. Superprocesses and plankton dynamics. in: Monte Carlo Simulation in Oceanography, Proc. of the 'Aha Huliko'a Hawaiian Winter Workshop, Univ. of Hawaii at Manoa, (1997), 121-128.

[3] D. Aldous. Deterministic and stochastic models for coalescence (aggregation and coagulation): a review of the mean-field theory for probabilists. Bernoulli, 5 (1999), 3-48.

[4] H. Amann, C. Walker. Local and global strong solutions to continuous coagulationfragmentation equations with diffusion. J. Differential Equations, 218 (2005), 159-186.

[5] M. Andersen. Spatial analysis of two-species interactions. Oecologia, 91 (1992), 134-140.

[6] O. Arino, R. Rudnicki. Phytoplankton dynamics. C. R. Biologie, 327 (2004), 961-969.

[7] J. Banasiak. On a non-uniqueness in fragmentation models. Math. Methods Appl. Sci., 25 (2002), 541-556.

[8] V.P. Belavkin, V.N. Kolokoltsov. On a general kinetic equation for many-particle systems with interaction, fragmentation and coagulation. R. Soc. Lond. Proc. Ser. A Math. Phys. Eng. Sci., 459 (2003), 727-748.

[9] F.E. Boyd, M. Cai, H. Han. Rate equation and scaling for fragmentation with mass loss. Phys. Rev. A, 41 (1990), 5755-5757.

[10] P.J. Clark, F.C. Evans. Distance to nearest neighbor as a measure of spatial relationships in populations. Ecology, 35 (1954), 445-453.

[11] H.E. Dam, D.T. Drapeau. Coagulation efficiency, organic-matter glues and the dynamics of particle during a phytoplankton bloom in a mesocosm study. Deep-Sea Research II, 42 (1995), 111-123.

[12] D. A. Dawson. Measure-valued Markov processes. École d'Été de Probabilités de SaintFlour XXI-1991, Lecture Notes in Math. 1541, Springer, Berlin, 1993.

[13] P. Donnelly, T.G. Kurtz. Particle representations for measure-valued population processes. Ann. Probab., 27 (1999), 166-205.

[14] R. L. Drake. A general mathematical survey of the coagulation equation. in: Topics in Current Aerosol Research (Part 2), G. M. Hidy and J. R. Brock (eds.), Pergamon Press, Oxford, 1972, 201-376. 
[15] E.B. Dynkin. An Introduction to Branching Measure-Valued Processes. CRM Monogr. Ser. 6, Amer. Math. Soc., Providence, RI, 1994.

[16] A. Eibeck, W. Wagner. Stochastic particle approximations for Smoluchowski's coagulation equation. Ann. Appl. Probab., 11 (2001), 1137-1165.

[17] N. el Saadi, O. Arino. A superprocess with spatial interactions for modelling the aggregation behavior in phytoplankton. submitted.

[18] N. el Saddi. Modélisation et étude mathématique de populations structurées par des variables aléatoires: Application à 'agrégation du phytoplancton. $\mathrm{PhD}$ thesis Université de $\mathrm{Pau}$ (2004).

[19] A. M. Etheridge. An introduction to superprocesses. Univ. Lecture Ser. 20, Amer. Math. Soc., Providence, RI, 2000.

[20] N. Fournier, J-S. Giet. On small particles in coagulation-fragmentation equations. J. Statist. Phys., 111 (2003), 1299-1329.

[21] Y.M. Ioannides, H.G. Overman. Spatial evolution of the US urban system. Journal of economic geography, 4 (2004), 131-156.

[22] S.N. Ethier, T.G. Kurtz. Markov Processes: Characterization and Convergence. Wiley, New York, 1986.

[23] S. Ghosal, S. Mandre.A simple model illustrating the role of turbulence on phytoplankton blooms. J. Math. Biol., 46 (2003), 333-346.

[24] S. Grosskinsky, C. Klingenberg, K. Oelschläger. A rigorous derivation of Smoluchowski's equation in the moderate limit. Stochastic Anal. Appl., 22 (2004), 113-141.

[25] G.A. Jackson. A model of the formation of marine algal flocks by physical coagulation processes. Deep-Sea Research, 37 (1990), 1197-1211.

[26] G.A. Jackson. Comparing observed changes in particle size spectra with those predicted using coagulation theory. Deep-Sea Research II, 42 (1) (1995), 159-184.

[27] V.N. Kolokoltsov. Hydrodynamic limit of coagulation-fragmentation type models of k-nary interacting particles. J. Statist. Phys., 115 (2004), 1621-1653.

[28] T.G. Kurtz. Particle representations for measure-valued population processes with spatially varying birth rates. in: Stochastic models (Ottawa, ON, 1998), CMS Conf. Proc. 26, Amer. Math. Soc., Providence, RI, 2000, 299-317.

[29] M. Lachowicz, D. Wrzosek. A nonlocal coagulation-fragmentation model. Applicationes Math., 27 (2000), 45-66. 
[30] P. Laurençot, S. Mischler. The continuous coagulation-fragmentation equations with diffusion. Arch. Ration. Mech. Anal., 162 (2002), 45-99.

[31] P. Laurençot, D. Wrzosek. From the nonlocal to the local discrete diffusive coagulation equations. Math. Models Meth. Appl. Sci., 12 (2002), 1035-1048.

[32] A.A. Lushnikov. From sol to gel exactly. Phys. Rev. Lett., 93 (2004), 198-302.

[33] S.R. Massel. Fluid Mechanics for Marine Ecologists. Springer-Verlag, Berlin, 1999.

[34] D. Morale, V. Capasso, K. Oelschläger. An interacting particle system modelling aggregation behavior: from individuals to populations. J. Math. Biol., 50 (2005), 49-66.

[35] J.R. Norris. Brownian coagulation. Commun. Math. Sci., 2 (2004), 93-101.

[36] J.R. Norris. Smoluchowski's coagulation equation: uniqueness, non-uniqueness and a hydrodynamic limit for the stochastic coalescent. Ann. Appl. Probab., 9 (1999), 78-109.

[37] K. Oelschläger. Limit theorems for age-structured populations. Ann. Probab., 18 (1990), 290-318.

[38] U. Passow, A. L. Alldredge. Aggregation of a diatom bloom in a mesocosm: The role of transparent exopolymer particles (TEP). Deep-Sea Research II, 42 (1995), 99-109.

[39] A. Pazy. Semigroups of Linear Operators and Applications to Partial Differential Equations. Appl. Math. Sci., 44, Springer, New York, 1983.

[40] H. Pretzsch. Analysis and modelling of spatial stand structures. Methodological considerations based on mixed beech-larch stands in Lower Saxony. Forest Ecology and Management, 97 (1997), 237-253.

[41] R. Rudnicki, R. Wieczorek. Fragmentation - coagulation models of phytoplankton. Bull. Pol. Acad. Sci. Math., in press.

[42] M. von Smoluchowski. Drei Vorträge über Diffusion, Brownsche Molekularbewegung und Koagulation von Kolloidteilchen. Phys. Z. ,17 (1916), 557-571, 585-599.

[43] W. Wagner. Explosion phenomena in stochastic coagulation-fragmentation models. Ann. Appl. Probab., 15 (2005), 2081-2112.

[44] W.R. Young, A.J. Roberts, G. Stuhne. Reproductive pair correlations and the clustering of organisms. Nature, 412 (2001), 328-331.

[45] W.R. Young. Brownian bugs and superprocesses. In: Mixing, Proc. of the 12th 'Aha Huliko'a Hawaiian Winter Workshop, University of Hawaii at Manoa, (2001), 123-129.

[46] R.M. Ziff, E. D. McGrady. Kinetics of polymer degradation. Macromolecules, 19 (1986), 2513-2519. 